\title{
Change blindness: Past, present, and future
}

\author{
Daniel J. Simons ${ }^{1}$ and Ronald A. Rensink ${ }^{2}$ \\ ${ }^{1}$ Psychology Department and Beckman Institute, University of Illinois, 603 E. Daniel Street, Champaign IL 61820, USA \\ ${ }^{2}$ Departments of Psychology and Computer Science, University of British Columbia, 2136 West Mall, Vancouver BC, V6T \\ 1Z4, Canada
}

\begin{abstract}
Change blindness is the striking failure to see large changes that normally would be noticed easily. Over the past decade this phenomenon has greatly contributed to our understanding of attention, perception, and even consciousness. The surprising extent of change blindness explains its broad appeal, but its counterintuitive nature has also engendered confusions about the kinds of inferences that legitimately follow from it. Here we discuss the legitimate and the erroneous inferences that have been drawn, and offer a set of requirements to help separate them. In doing so, we clarify the genuine contributions of change blindness research to our understanding of visual perception and awareness, and provide a glimpse of some ways in which change blindness might shape future research.
\end{abstract}

\section{Introduction}

The term 'change blindness' refers to the surprising difficulty observers have in noticing large changes to visual scenes [1,2]. Although failures to detect change have been studied for decades [3], recent work has brought change blindness out of the laboratory and into the realm of more typical perceptual experience. But although the often counterintuitive nature of change blindness has been the source of considerable insight into visual perception and awareness, it has also been the source of considerable confusion as to the inferences that can be legitimately drawn from it. Consequently, the results of these studies have sometimes been mischaracterized, leading to extreme and relatively unsupported claims, some of which havegained traction in the field. In rejecting these claims, some researchers have been tempted to summarily dismiss the entire field, essentially throwing out the baby with the bathwater.

The goal of this opinion article is to counter this potentially unfortunate trend by: (i) highlighting the new and important insights that have legitimately been obtained from recent work on change blindness; (ii) identifying common misconceptions about the kinds of inferences that can be drawn from it; (iii) delineating the constraints on such inferences so that future work will not fall prey to these misunderstandings; and (iv) describing some new ways in which change blindness can provide a useful tool for the study of attention, awareness, and visual perception.

Corresponding authors: Simons, D.J. (dsimons@uiuc.edu); Rensink, R.A. (rensink@psych.ubc.ca).

\section{Insights obtained from change blindness}

The recent surge of interest in change blindness followed decades of related work on the interplay between visual perception and memory [3]. For example, numerous previous studies showed that observers often fail to notice the displacement of a target dot or the change in shape of a line-drawn object if the change occurs during an eye movement (see [4] for an overview). This earlier work addressed many of the same theoretical issues revivified by more recent research, including limitations on memory capacity, links between attention and awareness, and the integration of information over time. However, an overarching theoretical framework linking these issues was never developed.

\section{Generality of effect}

The shift to a more general view of change blindness was triggered - at least in part - by the discovery in 1991 that observers often failed to notice large changes to photographs that were made during an eye movement [5]. For example, $50 \%$ percent of observers failed to notice when two cowboys sitting on a bench exchanged heads! These shocking results inspired others to examine whether similar failures could happen in other ways, and in the absence of eye movements. In one of these new paradigms - the 'flicker' task [1] - an original and modified scene alternate repeatedly, separated a brief blank display, until observers find the change. Observers eventually find most changes, but can take an astonishingly long time to do so, even for large changes. Similar results were found for changes made during eye blinks, 'mudsplats', and brief occlusions, as well as changes made gradually (see [3] for an overview). All these techniques (as well as those based on eye movements) involved a common element: they impaired localization of the motion signals that accompanied the change. This suggested that attention is needed for change perception, with change blindness resulting whenever the accompanying motion signals failed to draw attention [1].

Other studies discovered that these effects are even stronger when the changes are unexpected. For example, if an actor in a scene is changed during a shift in camera position, most observers do not notice, even if the actor is replaced by another person [6]. In fact, many observers fail to notice when a conversation partner is replaced by a different person in the middle of a real-life interaction [7,8]. The use of naturalistic stimuli in more recent work represented a signi- 
ficant departure from earlier research, one which helped bridge the gap between the simple stimuli usually found in the laboratory and the complex stimuli usually found in the real world. The counterintuitive finding of substantial change blindness under more naturalistic conditions strongly supports the view that it is not an artifact produced by artificial disruptions; rather, it is a general failure to retain and/or compare information from moment to moment. Moreover, this counterintuitiveness itself is of scientific interest; most people firmly believe that they would notice such large changes $[9,10]$. The practical ramifications of this 'change blindness blindness' are significant; for example, the mistaken belief that unexpected events always draw attention might help account for 'looked but didn't see' automobile accidents.

\section{Relation to attention}

Another important finding established by these newer studies is that attention is needed to see change. Changes to semantically central items are detected faster than changes elsewhere [1], even when the changes are of equal physical salience [11], suggesting that objects in a scene that preferentially receive attention are more likely to be encoded and compared. This finding inspired further insights into the nature of focused attention, the nature of visual short-term memory, and the relation between them. For example, although attention can be distributed to 4-5 items at a time [3], only a single change can be seen at any moment ('change simultagnosia') [12], indicating that the information from these items is pooled into a single collection point (or 'nexus'). Moreover, properties of the same kind compete for binding into a single integrated object, but properties of different kinds do not, suggesting that they are represented by parallel systems [13].

Interestingly, the connection between attention and awareness is not symmetrical: although attention is necessary for conscious change perception, it might not be sufficient. Changes to attended objects frequently go unnoticed $[14,15]$, particularly when the changes are unexpected. This suggests that object perception is highly dynamic - the properties consciously perceived at any moment are just those needed for the task at hand [15]. Such findings counter earlier claims that attention always binds features into a complete representation of an object [16-18].

Given the link between attention and awareness, change detection has also been used to measure the locus of attention, much as eye tracking can measure the locus of fixation. For example, Tse and colleagues asked subjects to search for a change in a large array, using successful detection to indicate the locus of attention [19]. Interestingly, detection was enhanced both at the location of a flashed cue and on the opposite side of fixation, indicating that attention is symmetrically distributed around the fixation point, something not previously suspected. This technique, in principle, could serve as an 'attention tracker,' providing a measure of covert attention independent of fixation. Moreover, it could potentially be used to assess not only what observers are attending to, but also what aspects of it (e.g. overall shape, color, particular parts).

\section{Insights into other visual processes}

These developments have in turn provided a novel way to explore perceptual organization and grouping processes. For example, studies using the flicker task suggest that items of similar color can be grouped into a single memory structure [12]. Change-detection failures also have been used to explore the role of scene context in guiding attention [20] and to provide an indication of foreground-background segmentation and re-segregation [21].

Given that attention is limited, our perception of dynamic events in a scene requires careful attention management [3]. Faster change detection implies earlier attention to the changed property, and an analysis of such 'attention scanpaths' can provide important insights into individual and group differences. For example, social drug users are more likely to detect changes to drug paraphernalia in photographs than are non-drug users [22] and American football experts are better able to spot meaningful changes to football scenes than are novices [23]. Change blindness can also help determine the mechanisms and strategies used by patients with various visual deficits [24,25].

Work on change blindness has also helped at an operational level, refining concepts which were not previously well articulated. For example, it forced a clarification of the distinction between motion perception, change perception, and difference detection, notions that often were conflated in earlier work: Motion perception is now more clearly understood as the detection of unorganized flow at a location, change perception as the detection of an ongoing transformation of a structured object, and difference perception as an inferential comparison of the current stimulus with traces in long-term memories [3]. These distinctions can be applied broadly, for example in distinguishing between the contributions of motion and change in the perception of facial expression [26].

Change detection research has also developed ways to explore possible non-attentional (and perhaps nonconscious) contributions to visual perception. Such processes might register the presence of a change before conscious perception, either via the possible [27] operation of implicit processes [14,28-30] or via other mechanisms such as 'mindsight' - the ability of some observers to experience a 'gut feeling' as to the presence of a change before they visually identify it [31]. This 'sensing' of a change might constitute a new perceptual mechanism revealed through the use of change blindness (but see [32]). Although such non attentional pathways are controversial, change blindness has inspired novel, testable hypotheses about the mechanisms of perception.

\section{Limits to what can be inferred from change blindness}

Unfortunately, these important advances have been clouded by the drawing of several overly strong conclusions. For example, earlier work on visual integration inspired the claim 
that our visual representations are sparse, incomplete, or absent altogether [33,34], and the striking blindness to large changes in scenes strengthened the appeal of this view. If true, this conclusion would require radical revisions of our way of thinking about perception, memory, and awareness [35]. However, the existence of change blindness does not on its own necessitate sparse representations - it could occur even with fairly detailed and complete visual representations of a scene [36-38]. Despite regular mention of the fact that representations might exist in the face of change blindness $[2,36]$, conclusions of absent representations have become increasingly prevalent[35], with conference sessions devoted to the idea (for example, at the 2004 Meeting of the Association for the Scientific Study of Consciousness). Many researchers who recognize the logical flaw have consequently been tempted to dismiss the entire line of research, thereby throwing out the good along with the bad.

In an attempt to counter these potentially unfortunate trends, we identify four 'requirements of scope', which delineate the possibilities that need to be ruled out before a blanket assertion of sparse (or absent) representations could
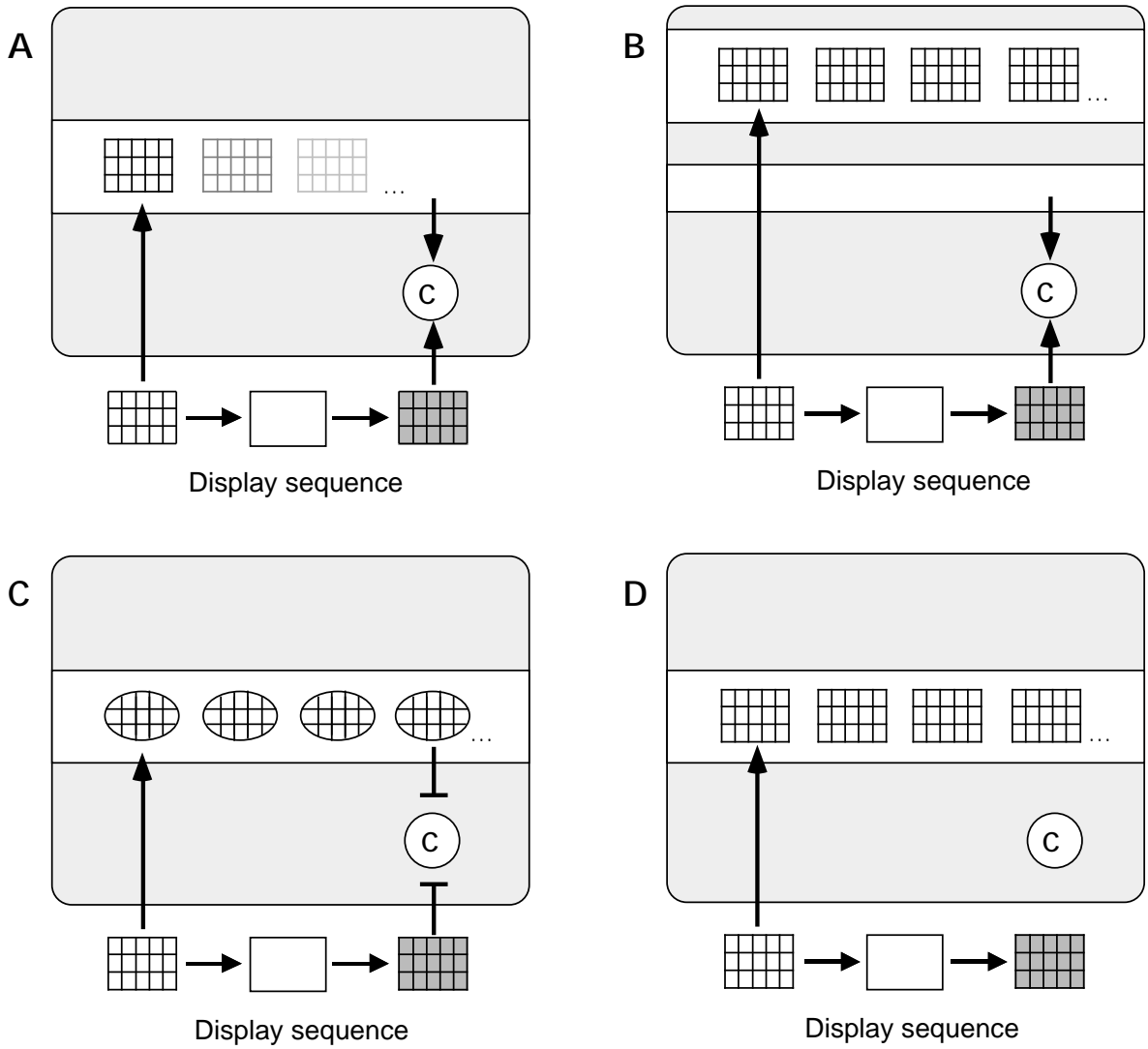

Figure 1. The consequences of failing to meet the requirements of scope. Each panel illustrates how information can be preserved in the face of change blindness if a requirement of scope is not met. The sequence of displays viewed by the observers appears below a gray box indicating what is retained from the initial display over time. ' $C$ ' indicates a comparison process and the arrows indicate what is compared. In (a), the representation is initially complete, but fades or is disrupted before it can be compared with the post-change scene. When the comparison eventually occurs, the initial representation is gone, so change blindness ensues. Change blindness says nothing about such short-lived representations. In (b), the representation is complete, but it does not enter the pathway used for the comparison process, so change blindness ensues. In (c), the representation is detailed but in a format that precludes successful change detection. In (d), the representation is complete and detailed, but the comparison process fails and the change is not detected. Note that in each of the four cases, the representation of the first display could be complete and detailed (at least momentarily). Even if the representations are not complete and detailed, the logic underlying the requirements of scope and the limitations they place on inferences from change blindness still apply. 


\section{Box 1. Perception versus inference}

Failure to recognize the requirements of scope sometimes leads to the erroneous conclusion that all visual representations are sparse or absent. Yet, similar failures can also lead to illegitimate conclusions that overestimate the completeness of the representations underlying conscious 'here-and-now' visual experience [37].

Observers can sometimes detect a change when they later refixate a changed item, implying that some information about the pre change object was stored somewhere. But the inference of a change at some point in the past does not imply that the observer also perceived the change. Indeed, these processes might use entirely different mechanisms and representations [3]. For example, many observers fail to notice when a building in a photograph gradually disappears over the course of $12 \mathrm{~s}$. However, upon viewing the initial state of the photograph again, they then realize that the building is missing [47]. They did not have a visual experience of it changing; rather, they realized that it was different, possibly on the basis of long-term memory.

Although both mechanisms involve preserved information, it is important not to conflate them; otherwise, there is a risk of inappropriately ascribing the capacity of the long-term mechanisms presumably used for inference to the shorter-term mechanisms used for change perception, inflating estimates of the information they contain. Of course, more complete and detailed 'pixilated' representations might be involved in change perception [37,38], but the existence of these does not logically follow from existing work. This inference would require establishing that only the short-term mechanisms underlying change perception were being tested, with no direct contributions from longer-term structures.

possibility. (Interestingly, the presence of multiple pathways can also lead to an overestimate of the information available for change perception; see Box 1.)

(3) Evidence must eliminate the possibility that such representations exist, but are in a format that cannot be used for change perception. In other words, evidence must show that whatever can be seen, can be seen to change. If this requirement is not met, conclusions must be limited to those representations that subserve the perception of change, rather than perception in general. Tests not involving comparison (e.g. information integration across saccades; see [34]) could potentially examine such representations. But change blindness can say nothing about the nature of representations that cannot be compared.

(4) Evidence must eliminate the possibility that such representations exist in an appropriate format and pathway, but the comparison operation is not applied (even though it could be). For example, if the changedetection task is time-limited and the comparison mechanism slow, observers might not be able to compare the changed region in time $[36,38,44]$. And even with sufficient time, these mechanisms might not be engaged by tasks that do not compel their use [45]. Consequently, change blindness cannot rule out the existence of representations that were not accessed.

In summary, the failure to detect change does not imply the absence of a representation unless all the requirements of scope are met. Given that no studies have done this, extant work on change blindness can say nothing about the representations that subserve the static aspects of vision - it can only reveal limits on the representations that subserve the conscious perception of dynamic change. In fact, mounting evidence suggests that some information is preserved even when observers fail to detect changes $[37,38,44]$. And theoretical frameworks based on change blindness studies have long asserted that at least some information concerning the static aspect of a scene must be maintained (over both short and long time scales) if scene perception is to operate effectively [40].

\section{The future of change blindness research}

Although there are limits to some of the conclusions that can be drawn from change blindness, considerable potential remains. Change blindness has already contributed to our understanding of various mechanisms of visual perception, including those that are central to our conscious experience of vision. It has extended the reach of empirical techniques beyond the traditional boundaries of cognition and perception research, providing a new way to explore individual differences, expertise, and even cultural differences [46]. And it may even provide new ways of studying aspects of individual experience that have traditionally been difficult to investigate (e.g. implicit perception; see also Box 2).

Change blindness has contributed to a resurgence of the study of scene perception, and in particular, of the dynamics that underlie it. More broadly, change detection and change perception can be considered special cases of event perception, and the concepts and techniques developed in this literature could become useful tools for understanding the perception of dynamic events more generally. Such an approach holds promise for several reasons. First, studies of change blindness use a wide variety of stimuli, from controlled displays to real-world interactions. Second, models based on

\section{Box 2. Outstanding issues and questions}

- Can observers detect a change even if they fail to perceive it consciously? More precisely, does change perception require a conscious comparison mechanism [27]? If not, are there alternative routes to change detection $[29,30]$ ? Such alternative routes would suggest that attention might not be necessary for change detection (or perhaps that it is necessary only for conscious perception of change).

- How detailed are our visual representations? Earlier frameworks posited that preserved representations were relatively sparse, containing for example less than $10 \%$ of the information (in terms of bits) in the incoming image [48]. However, the amount of preserved information might be much higher [38]. New research could address this issue by fully meeting the requirements of scope, by clarifying what is meant by 'detail', and by using converging measures from tasks and methods not based on change perception.

- Do people experience the presence of a change before they can explicitly localize and identify it? The evidence for mindsight suggests that it is an experiential state quite different from the 'picture' that usually accompanies the pick-up of visual information. Does mindsight constitute evidence for a previously unknown perceptual mechanism [31]? Or can it be accounted for by individual differences in the criteria used to report a change [32]?

- To what extent and in what manner do long-term representations (visual or otherwise) contribute to change-detection performance? When long-term memory does contribute, are the mechanisms responsible qualitatively different from when change detection happens via immediate perception? Does the comparison of long term information differ from the immediate perception of change [[3]? Are the contents of the longer-terms stores ever transferred to the shorter-term mechanisms? 
change blindness already address the nature of object representations, allowing a natural extension to events. Third, change-blindness research inherently concerns scene perception over time, something that most models of object recognition do not. As such, there is potential to extend the concepts and techniques developed in this field of research to more complex events, thereby enabling the successful exploration of a world of interesting new phenomena.

\section{References}

1. Rensink, R.A. et al. (1997) To see or not to see: The need for attention to perceive changes in scenes. Psychol. Sci. 8, 368-373

2. Simons, D.J. and Levin, D.T. (1997) Change blindness. Trends Cogn. Sci. 1, 261-267

3. Rensink, R.A. (2002) Change detection. Annu. Rev. Psychol. 53, 245-277

4. Bridgeman, B. et al. (1994) A theory of visual stability across saccadic eye movements. Behav. Brain Sci. 17, 247-292

5. Grimes, J. (1996) On the failure to detect changes in scenes across saccades. In Perception (Vancouver Studies in Cognitive Science, Vol. 5) (Akins, K., ed.), pp. 89-110, Oxford University Press

6. Levin, D.T. and Simons, D.J. (1997) Failure to detect changes to attended objects in motion pictures. Psychon. Bull. Rev. 4, 501-506

7. Simons, D.J. and Levin, D.T. (1998) Failure to detect changes to people in a real-world interaction. Psychon. Bull. Rev. 5, 644-649

8. Levin, D.T. et al. (2002) Memory for centrally attended changing objects in an incidental real-world change detection paradigm. Br. J. Psychol. 93, 289-302

9. Levin,D.T.et al. False predictions about the detectability of unexpected visual changes: The role of beliefs about attention, memory, and the continuity of attended objects in causing change blindness blindness. Conscious. Cogn. (in press)

10. Levin, D.T. et al. (2000) Change blindness blindness: The metacognitive error of overestimating change-detection ability. Vis. Cogn. 7, 397-412

11. Kelley, T.A. et al. (2003) Effects of scene inversion on change detection of targets matched for visual salience. J. Vis. 3, 1-5

12. Rensink, R.A. (2002) Failure to see more than one change at a time. J. Vis. 2, 245a

13. Wheeler, M.E. and Treisman, A. (2002) Binding in short-term visual memory. J. Exp. Psychol. Gen. 131, 48-64

14. Williams, P. and Simons, D.J. (2000) Detecting changes in novel, complex three-dimensional objects. Vis. Cogn. 7, 297-322

15. Triesch, J. et al. (2003) What you see is what you need. J. Vis. 3, 86-94

16. Luck, S.J. and Vogel, E.K. (1997) The capacity of visual working memory for features and conjunctions. Nature 390, 279-281

17. Treisman, A.M. and Gelade, G. (1980) A feature-integration theory of attention. Cogn. Psychol. 12, 97-136

18. Kahneman, D. et al. (1992) The reviewing of object files: Objectspecific integration of information. Cogn. Psychol. 24, 175-219

19. Tse, P.U. et al. (2003) Attentional enhancement opposite a peripheral flash revealed using change blindness. Psychol. Sci. 14, 91-99

20. Jiang, Y. et al. (2004) Perceptual grouping in change detection. Percept. Psychophys. 66, 446-453

21. Landman, R. et al. (2004) The role of figure-ground segregation in change blindness. Psychon. Bull. Rev. 11, 254-261

22. Jones, B.T. et al. (2003) A flicker paradigm for inducing change blindness reveals alcohol and cannabis information processing biases in social users. Addiction 98, 235-244

23. Werner, S. and Thies, B. (2000) Is 'change blindness' attenuated by domain-specific expertise? An expert-novices comparison of change detection in football images. Vis. Cogn. 7, 163-174
24. Pisella, L. et al. (2004) Impaired working memory for location but not for colour or shape in visual negelct: A comparison of parietal and nonparietal lesions. Cortex 40, 379-390

25. Rutkowski, J.S. et al. (2003) Change detection is impaired in children with dyslexia. J. Vis. 3, 95-105

26. Ambadar, Z. et al. Deciphering the enigmatic face: The importance of facial dynamics in interpreting subtle facial expressions. Psychol. Sci. (in press)

27. Mitroff, S.R. et al. (2002) The siren song of implicit change detection. J. Exp. Psychol. Hum. Percept. Perform. 28, 798-815

28. Fernandez-Duque, D. and Thornton, I.M. (2000) Change detection without awareness: Do explicit reports underestimate the representation of change in the visual system? Vis. Cogn. 7, 323-344

29. Thornton, I.M. and Fernandez-Duque, D. (2000) An implicit measure of undetected change. Spat. Vis. 14, 21-44

30. Fernandez-Duque, D. et al. (2003) Representation of change: Separate electrophysiological markers of attention, awareness, and implicit processing. J. Cogn. Neurosci. 15, 491-507

31. Rensink, R.A. (2004) Visual sensing without seeing. Psychol. Sci. 15, 27-32

32. Simons, D.J. et al. Visual sensing is seeing: Why 'mindsight', in hindsight, is blind. Psychol. Sci. (in press)

33. O'Regan, J.K. (1992) Solving the 'Real' mysteries of visual perception: The world as an outside memory. Can. J. Psychol. 46, 461-488

34. Irwin, D.E. (1991) Information integration across saccadic eye movements. Cogn. Psychol. 23, 420-456

35. O'Regan, J.K. and Noe", A. (2001) A sensorimotor account of vision and visual consciousness. Behav. Brain Sci. 24, 939-1031

36. Simons, D.J. (2000) Current approaches to change blindness. Vis. Cogn. 7, 1-15

37. Hollingworth, A. and Henderson, J.M. (2002) Accurate visual memory for previously attended objects in natural scenes. J. Exp. Psychol Hum. Percept. Perform. 28, 113-136

38. Hollingworth, A. (2003) Failures of retrieval and comparison constrain change detection in natural scenes. J. Exp. Psychol. Hum. Percept. Perform. 29, 388-403

39. Becker, M.W. et al. (2000) The role of iconic memory in change detection tasks. Perception 29, 273-286

40. Rensink, R.A. (2000) The dynamic representation of scenes. Vis. Cogn. 7, 17-42

41. Goodale, M.A. and Milner, A.D. (1992) Separate visual pathways for perception and action. Trends Neurosci. 15, 20-25

42. Goodale, M.A. et al. (1986) Large adjustments in visually guided reaching do not depend on vision of the hand or perception of target displacement. Nature 320, 748-750

43. Bridgeman, B. et al. (1979) Relation between cognitive and motororiented systems of visual position perception. J. Exp. Psychol. Hum Percept. Perform. 5, 692-700

44. Mitroff, S.R. and Simons, D.J. Nothing compares two views: Change blindness results from failures to compare retained information. Percept. Psychophys. (in press)

45. Simons, D.J. and Mitroff, S. (2001) The role of expectations in change detection and attentional capture. In Vision and Attention (Harris, L.R. and Jenkin, M., eds.), pp. 189-207, Springer-Verlag

46. Nisbett, R.E. and Masuda, T. (2003) Culture and point of view. Proc. Natl. Acad. Sci. U. S. A. 100, 11163-11170

47. Simons, D.J. et al. (2000) Change blindness in the absence of a visual disruption. Perception 29, 1143-1154

48. Rensink, R.A. (2000) Seeing, sensing, and scrutinizing. Vision Res. 40, 1469-1487 